\title{
Voriconazole Induced Periostitis in a Patient with ANCA Vasculitis: A Case Report
}

\author{
Hafiz Bajwa*, Chun-Ruh Ng, Azhar Abdullah, Sadia Aslam, Paul 0’Connell \\ Department of Rheumatology, Beaumont Hospital, Dublin, Ireland \\ Email: ^hybajwa@hotmail.com
}

How to cite this paper: Bajwa, H., Ng, C.-R., Abdullah, A., Aslam, S. and O'Connell, P. (2019) Voriconazole Induced Periostitis in a Patient with ANCA Vasculitis: A Case Report. Open Access Library Journal, 6: e5789.

https://doi.org/10.4236/oalib.1105789

Received: September 16, 2019

Accepted: October 11, 2019

Published: October 14, 2019

Copyright $\odot 2019$ by author(s) and Open Access Library Inc.

This work is licensed under the Creative Commons Attribution International License (CC BY 4.0).

http://creativecommons.org/licenses/by/4.0/

\begin{abstract}
A 62 year-old gentleman with underlying ANCA vasculitis was admitted to hospital for severe musculoskeletal pain involving both shoulders, hips and hands. There was a history of long term usage of anti-fungal medication-voriconazole for his Aspergillus fumigatus lung infection as treatment and prophylaxis. Clinically the range of movement of both shoulders and hips was restricted with the presence of digital swelling of both hands. His imaging findings noted the presence of periosteal reaction involving shoulders and hips in addition to increased radiotracer uptake on his bone scan. A diagnosis of voriconazole induced periostitis was made and discontinuation of therapy led to rapid clinical improvement.
\end{abstract}

\section{Subject Areas}

Rheumatology

\section{Keywords}

Vasculitis, Voriconazole, Periostitis

\section{Introduction}

Voriconazole is a broad spectrum triazole derivative of fluconazole, the second generation antifungal agent with its function to interrupt cell membrane and terminate fungal growth by restraining the cytochrome P450 dependent enzyme 14-alpha-sterol demethylase [1]. Aspergillus fumigatus was previously regarded as a weak pathogen for many decades, which is now transformed into the commonest fungal infection globally owing to increase in the number of immunosuppressed patients dramatically. Without prompt treatment, the mortality rate can be high. However, long term usage of voriconazole can create undesirable side effects for patients and one of them is bone pain secondary to periostitis. 
Periostitis is a nonspecific imaging finding and its clinical entity comprises bone pain due to inflammation of the periosteum. Voriconazole was reported as the only member in the azole group that was capable to induce periostitis due to its trifluorinated compound and this supported the association between elevated plasma fluoride level and skeletal presentation [2].

\section{Case Report}

A 62 year-old gentleman was admitted to emergency department in January 2016 with 2 months history of severe pain and limited range of movement involving both shoulders, hips and hands. There was no rash, no respiratory symptoms or neurological involvement. He denied constitutional symptoms. On further history, he was diagnosed to have ANCA associated vasculitis complicated with crescentic glomerulonephritis and bowel perforation in February 2015. Subsequently, he was treated with intravenous methylprednisolone, cyclophosphamide and plasmapheresis as induction therapy for his active vasculitis. His vasculitis was in remission with intravenous Rituximab as maintenance therapy. During his hospitalisation, he developed intraabdominal sepsis and chronic aspergillus pneumonitis and he was managed with long term anti-fungal voriconazole therapy. His medications upon discharged were voriconazole $300 \mathrm{mg} \mathrm{BD}$ (long term prophylaxis), prednisolone $30 \mathrm{mg}$ daily and aspirin $75 \mathrm{mg}$ daily.

Clinical examination revealed he was pink, no rash with stable vital signs. There was no lymph node felt. His cardiovascular, respiratory, abdomen and central nervous system examinations were unremarkable. Muskuloskeletal examination revealed presence of tender digital swelling of both hands with no clubbing seen. There was reduced range of movement in hands and left shoulder. Hips examination noted presence of flexion contracture with limited range of movement and his right hip was affected more compared to the left.

Laboratory analysis showed normochromic normocytic anemia with a haemoglobin of $10.5 \mathrm{~g} / \mathrm{dl}, \mathrm{MCV} 81 \mathrm{fl}$ and white blood cell of 13,000 cells/ul. The inflammatory markers erythrocyte sedimentation rate (ESR) and C-reactive protein (CRP) were $73 \mathrm{~mm} / \mathrm{h}$ and $29 \mathrm{mg} / \mathrm{L}$ respectively. His serum alkaline phosphatase was raised to $412 \mathrm{U} / \mathrm{L}$ and plasma fluoride level was $278 \mu \mathrm{g} / \mathrm{L}$ (NR < $50 \mu \mathrm{g} / \mathrm{L})$.

Conventional radiograph of both shoulders, pelvis and left knee showed exuberant bilateral periosteal reaction involving all bones more prominent in the shoulders girdles, pelvis and hips [Figures 1-4]. A nuclear medicine bone scan demonstrated increased radiotracer uptake over both shoulders [Figure 5].

A diagnosis of voriconazole induced periostitis deformans was made after gathering all information and oral antifungal voriconazole was discontinued immediately. He reported significant clinical improvement thereafter.

\section{Discussion}

Voriconazole induced periostitis is a rare complication of long term usage of 


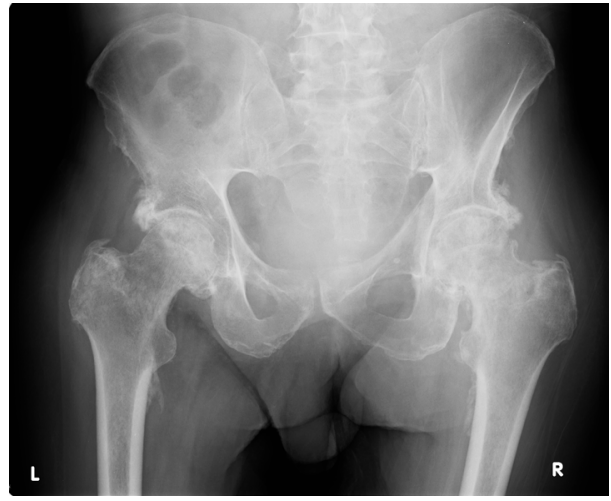

Figure 1. Demonstrates multifocal, dense, irregular periosteal new bone formation along the medial aspect of left humeral neck, the medial proximal right femur and both acetabulums area.

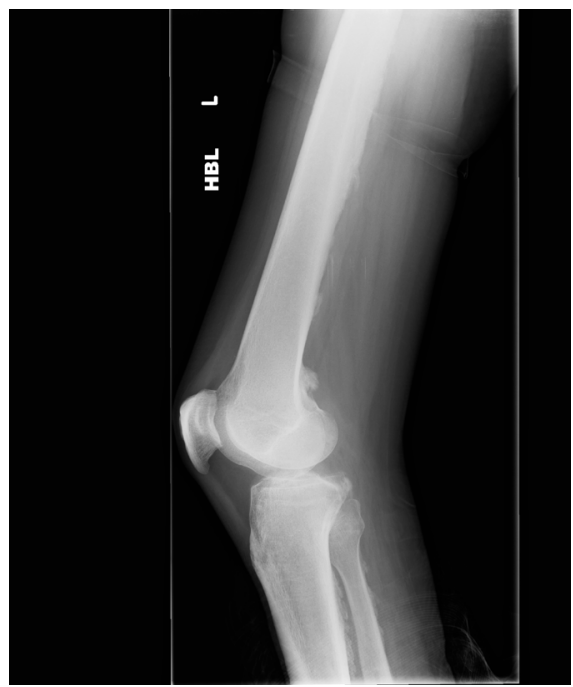

Figure 2. Illustrates multifocal periosteal reaction along the diaphysis of left femur and tibia fibula region.

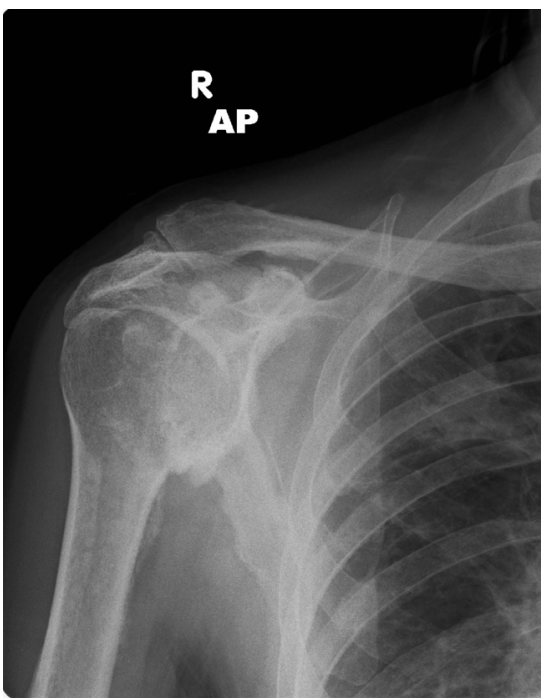

Figure 3. Illustrates nodular periostitis along the clavicles and scapula area. 


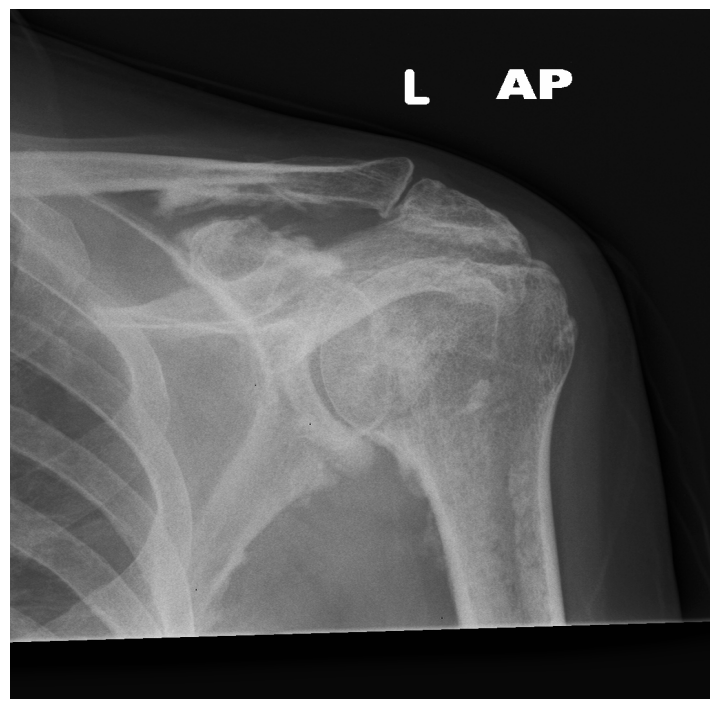

Figure 4. Illustrates nodular periostitis along the clavicles and scapula area.

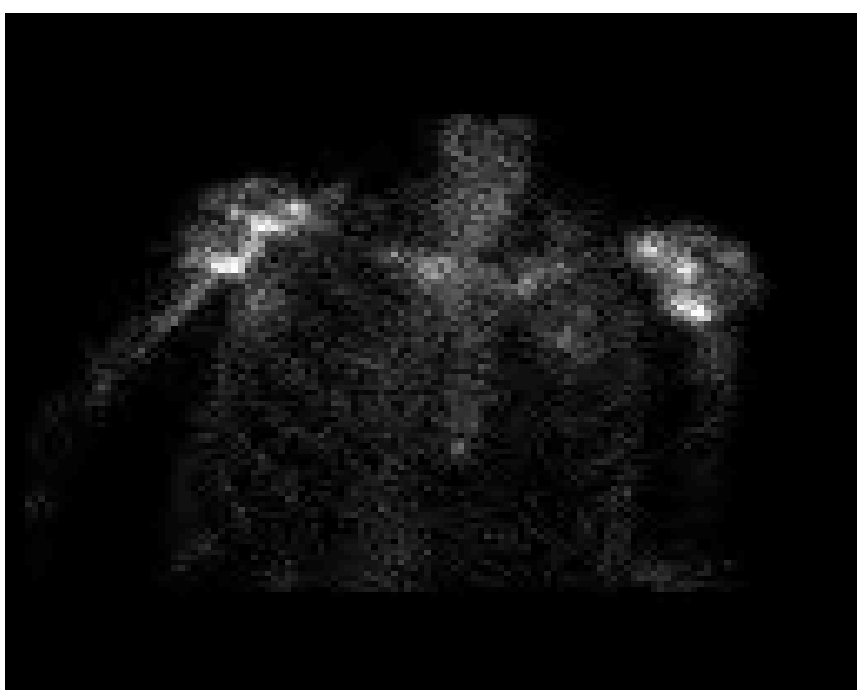

Figure 5. Demonstrates increase radionuclide uptake at both shoulders and clavicles.

antifungal therapy and was well reported in patients with solid organ transplantation who were on long term treatment few years ago [3]. Our patient was presented with periostitis after 10 months usage of voriconazole as treatment and prophylaxis when he developed chronic aspergillus pneumonia infection as a consequence of long term usage of immusuppressive agent for his ANCA vasculitis. He was admitted to emergency department for chronic bone pain with functional limitation due to painful periostitis. His musculoskeletal $\mathrm{x}$ rays noted typical findings of nodular and fluffy periosteal reaction which was asymmetrical in distribution. This assisted us to differentiate from hypertrophic osteoarthropathy which appeared more symmetrical with linear distribution [3] [4]. Besides, variconazole induced periostitis more commonly involve clavicle, scapula, acetabulum as showed in our patient [4] [5]. In addition to this, his serum alkaline phosphatase and fluoride plasma level were both raised markedly 
further confirmed his diagnosis of voriconazole induced periostitis. The proposed mechanism behind this reaction was the integration of fluoride into bone structure named fluorosis and this will stimulate osteoblasts activity, hence increase serum alkaline phosphatase and promote bone formation [6] [7].

Our patient was diagnosed to have variconazole induced periostitis deformans based on the constellation of his clinical history, presentation and laboratory analysis of elevated of serum alkaline phosphatase and fluoride levels, fluffy periosteal reaction on radiographs and multiple areas of uptake on bone scintigraphy formed the classical features after excluding infection and malignancy [3] [4].

Painful periostitis is a rare complication of chronic usage of voriconazole therapy and It had been described by Wang et al. in his patients who underwent solid organ transplantation and by Gerber et al. in patients with underlying hematologic malignancies [3] [6]. There is one case report published in 2014 documented a case of voriconazole induced periostitis in a patient with underlying overlap syndromes [8]. All cases reported were noted to have typical periostitis on bone scan and plain radiograph in conjunction with severe bone pain, lack of finger clubbing and elevated serum alkaline phosphatase level. These findings resolved followed by voriconazole discontinuation. This reversible response was well documented by Gerber B. et al. in his study of 43 patients with hematologic malignancies 2012 [6].

\section{Conclusion}

The diagnosis of voriconazole induced periostitis requires awareness of the disease in view of its rarity and varied clinical findings and rapid cessation of therapy led to clinical improvement in almost all patients. The risk factor for our patient to develop fluorosis is chronic usage of voriconazole therapy, which indicates the importance to obtain a good drug history for diagnosis.

\section{Support}

We declared no financial support involvement for the work in the manuscript.

\section{Consent}

Written informed consent was obtained from the patient for the publication of this case report and accompanying images.

\section{Conflicts of Interest}

We declared no conflict of interest with regard to the work.

\section{References}

[1] Scott, L.J., et al. (2007) Drugs. Voriconazole: A Review of Its Use in the Management of Invasive Fungal Infections.

[2] Tailor, T.D. and Richardson, M.L. (2015) Case 215, Voriconazole-Induced Periosti- 
tis. Radiology, 274, 930-935.

[3] Wang, T.F., Wang, T., Altman, R., Eshaghian, P., Lynch, J.P., Ross, D.J., Belperio, J.A., Weigt, S.S., Saggar, R., Gregson, A., Kubak, B. and Saggar, R. (2009) Periostitis Secondary to Prolonged Voriconazole Therapy in Lung Transplant Recipients. American Journal of Transplantation, 9, 2845-2850. https://doi.org/10.1111/j.1600-6143.2009.02837.x

[4] Chen, L. and Mulligan, M.E. (2011) Medication-Induced Periostitis in Lung Transplant Patients: Periostitis Deformans Revisited. Skeletal Radiology, 40, 143-148. https://doi.org/10.1007/s00256-010-0997-y

[5] Lustenberger, D.P., Granata, J.D. and Scharschmidt, T.J. (2011) Periostitis Secondary to Prolonged Voriconazole Therapy in a Lung Transplant Recipient. Orthopedics, 34, e793-e796. https://doi.org/10.3928/01477447-20110922-35

[6] Gerber, B., Guggenberger, R., Fasler, D., et al. (2012) Reversible Skeletal Disease and High Fluoride Serum Levels in Hematologic Patients Receiving Voriconazole. Blood, 120, 2390-2394. https://doi.org/10.1182/blood-2012-01-403030

[7] Lindsay, R. (1990) Fluoride and Bone-Quantity versus Quality. New England Journal of Medicine, 322, 845-846. https://doi.org/10.1056/NEJM199003223221210

[8] Hirota, K., Yasoda, A., Fujii, T. and Inagaki, N. (2014) Voriconazole-Induced Periostitis in a Patient with Overlap Syndromes. BMJ Case Reports, 2014, bcr2013203485. https://doi.org/10.1136/bcr-2013-203485 